\title{
Mapping the Disparities of Community Resilience to Natural Hazards in the United States
}

\author{
Lei Zou ${ }^{\text {a, }}$ * \\ a Department of Geography, Texas A\&M University, lzou@tamu.edu \\ * Corresponding author
}

Keywords: Disaster Resilience, Natural Hazards, Geographical Disparities, Geo-visualization, Sustainability

\begin{abstract}
:
Due to climate change and population growth in hazard-prone areas, human communities face increasing threats of natural disasters and significant socioeconomic consequences. Under the same strength of hazard, communities with various social-environmental conditions may endure different levels of disturbance and present diverse recovery patterns. These damage and recovery disparities can be attributed to the abilities of communities to "bounce back" from and adapt to adverse events, which are referred to as community resilience. Evaluating community resilience and identifying key factors that play a role in mitigating disaster impacts is critical for locating vulnerable social groups and helping decisionmakers formulate strategies to enhance resilience. Despite the robust literature on resilience assessment tools and metrics, most models were developed for specific regions or disaster types, defined resilience differently, and lacked empirical validation. Developing theoretically sound, empirically validated, and easy-to-implement frameworks to evaluate the disparities of resilience across communities facing different types of disasters and guide decision-making for resilience improvement remains challenging.
\end{abstract}

To address the challenge, this research developed a novel framework, the Improved Resilience Inference Measurement (IRIM) model, for community resilience assessment, and applied the model to map the disparities of community resilience to natural hazards in the United States. IRIM model defines community resilience by three dimensions, disaster threat, damage, and recovery, to denote two relationships: vulnerability and adaptability (Figure1). If a community has a high hazard threat but sustains low damage, then the community is considered to have low vulnerability. Similarly, if a community sustains serious damage but has a favorable recovery (e.g., return of population or economy), then the community is considered to have high adaptability. Community resilience is measured based on the two relationships in three steps: (1) indexes representing the three dimensions in each community are selected and calculated; (2) classification analysis is used to group the communities into multiple resilience categories based on each community's vulnerability and adaptability; and (3) machine learning and statistical methods are applied to identify the significant socialenvironmental variables that best characterize each resilience category.

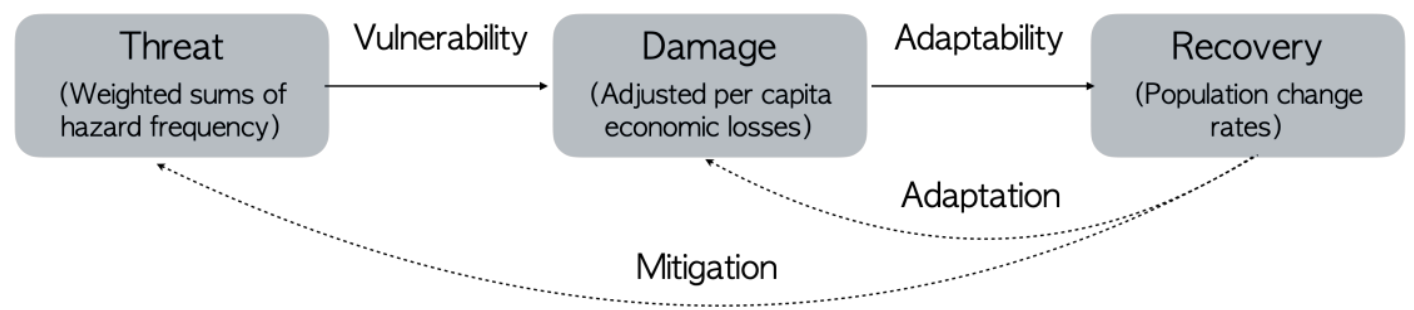

Figure 1. The Improved Resilience Inference Measurement (IRIM) model framework.

To map the county-level resilience to natural hazards in the United States during 2000-2010, we first computed the normalized disaster threat, damage, and recovery of all counties in the United States during the ten years. The threat was calculated as the weighted sums of hazard frequency, and the weights were the average daily per capita economic losses caused by each disaster type (Figure 2). The damage was defined as the adjusted cumulative per capita economic losses in each county during the ten-year period. The recovery was represented by population stability and calculated as population change rates. Second, we used the K-means clustering to classify U.S. counties into three vulnerability groups and three adaptability groups, resulting in a total of nine resilience categories. Finally, the discriminant analysis was conducted to identify resilience drivers and reveal each resilience level's social and environmental characteristics. 

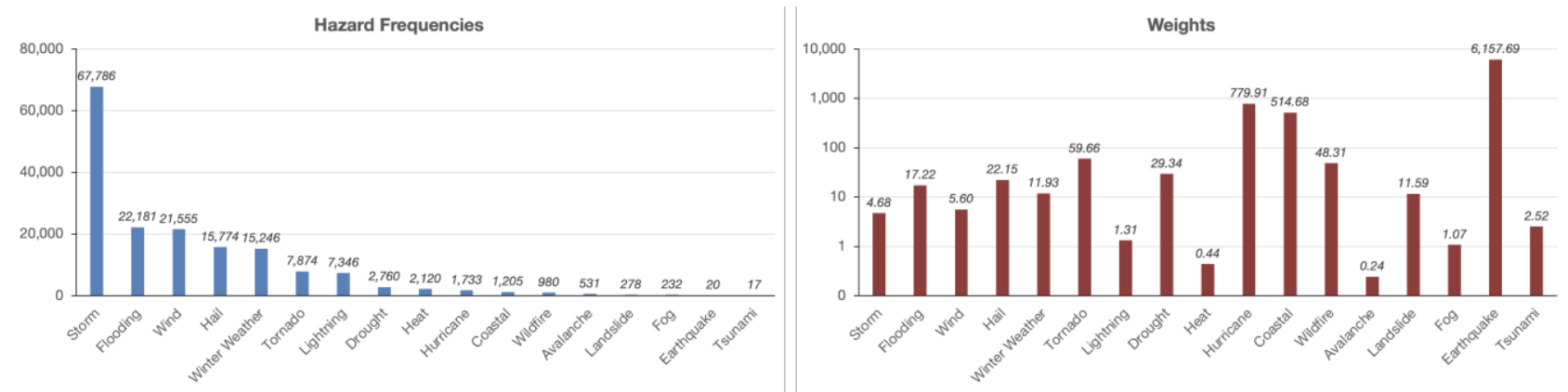

Figure 2. Frequencies and computed weights of the major types of disasters in the United States, 2000-2010.

The geographical disparities of the ten-year disaster threat, damage, recovery, and resilience in the United States are shown in Figure 3. Counties in Southern California, Oklahoma, and Hawaii, along the Gulf of Mexico, and near the eastern coastal line received more threats from natural hazards than inland communities. Meanwhile, counties in Louisiana and Mississippi suffered significantly greater damage than other counties. The recovery speed in the central United States was generally slower than in coastal regions. High resilience counties are concentrated in states along the western and eastern coastlines and northeastern Texas, whereas most counties in the North-Central U.S. have low resilience levels.
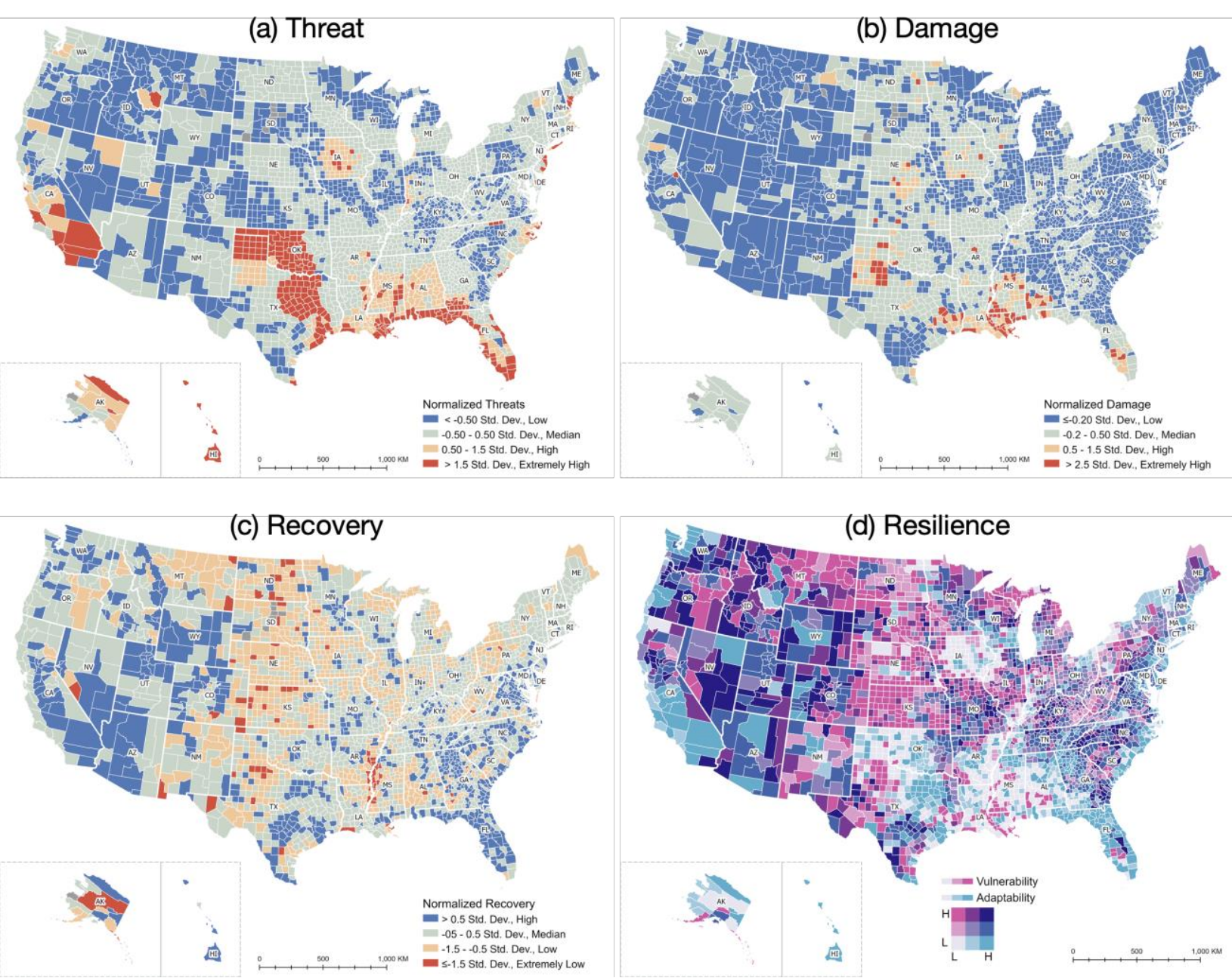

Figure 3. Normalized exposure, damage, recovery, and resilience levels during 2000-2010 in the United States.

The proposed resilience assessment framework is innovative and valuable for comparing community resilience across space and time. The analysis results reflect the geographical disparities of U.S. communities' ability to adapt, re-organize, and retain functions through multiple hazards. The discriminant functions derived can monitor the temporal dynamics of resilience levels in the U.S. counties and estimate other study areas' resilience levels using the same set of variables. The identified mechanisms between resilience capacities and driving forces could guide policymakers on where and how investments in intervention strategies may make a difference in resilience improvement. 